\title{
Molecular Design of Nickel Phthalocyanine@Nanocarbon Hybrid Single-atom Catalyst for Active and Stable Electroreduction of $\mathrm{CO}_{2}$
}

Zisheng Zhang ${ }^{\mathrm{a},+}$, Yang-Gang Wang ${ }^{\mathrm{a}^{*}}$

Department of Chemistry, Southern University of Science and Technology, Shenzhen 518055, China

${ }^{*}$ Correspondence should be addressed to:

wangyg@sustech.edu.cn

${ }^{+}$Current address: Department of Chemistry and Biochemistry, University of California, Los Angeles, 607 Charles E. Young Drive East, Los Angeles, California 90095, United States 


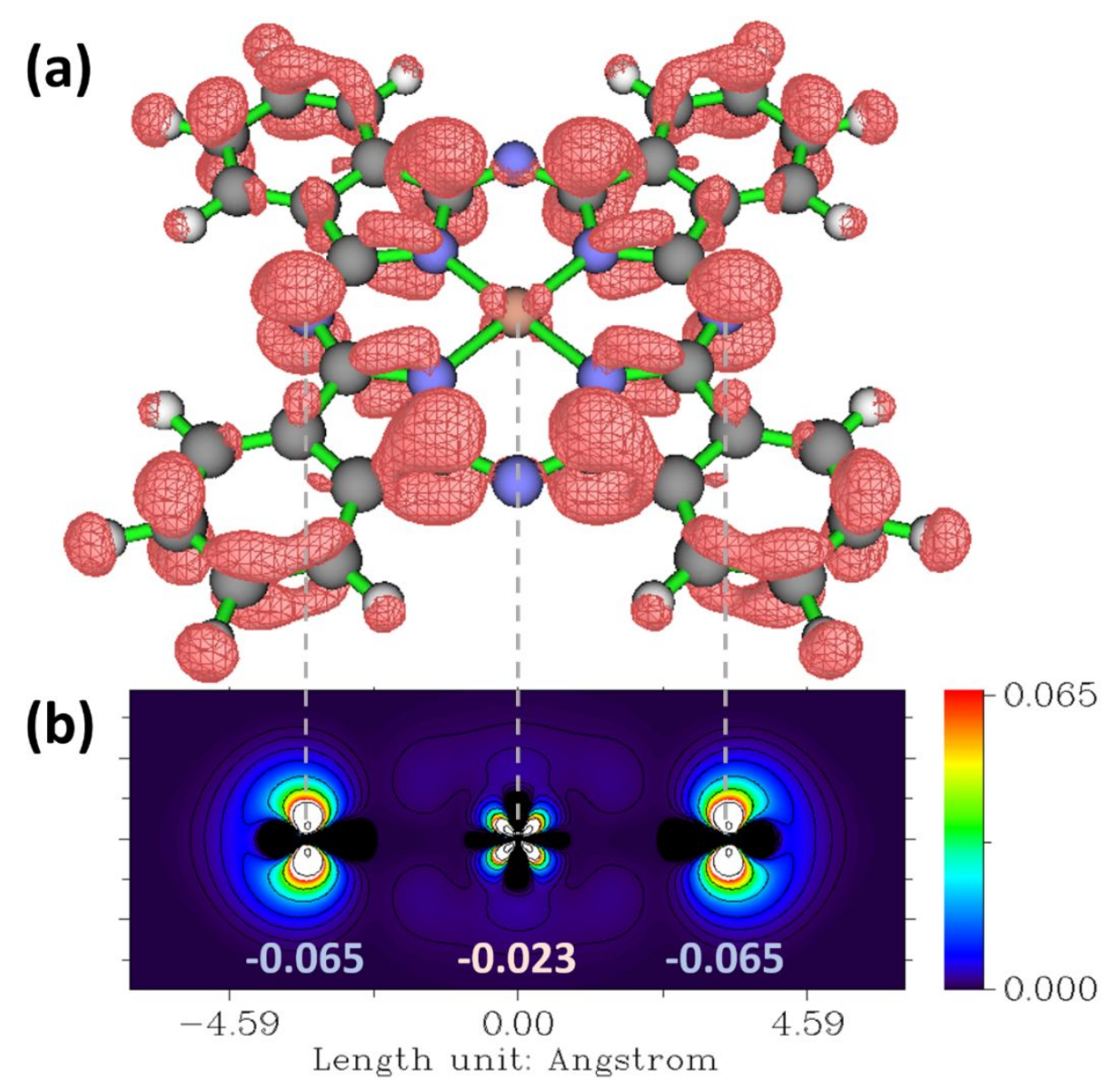

Figure S1. (a) Electron density map of NiPc after being reduced to [NiPc]-, with red isosurface showing the relative increase compared to neutral NiPc. Color code: $\mathrm{H}-$ white, $\mathrm{C}-$ grey, $\mathrm{N}-$ blue, $\mathrm{O}$ - red, $\mathrm{Ni}$ - brown. (b) Cross section of the electron density increment at $\mathrm{y}=0$ with colormap and contours. The change in Mulliken charges of the $\mathrm{Ni}$ (middle, in light red) and $\mathrm{N}$ atoms (left and right, in light blue) upon reduction are labeled under the corresponding regions. 


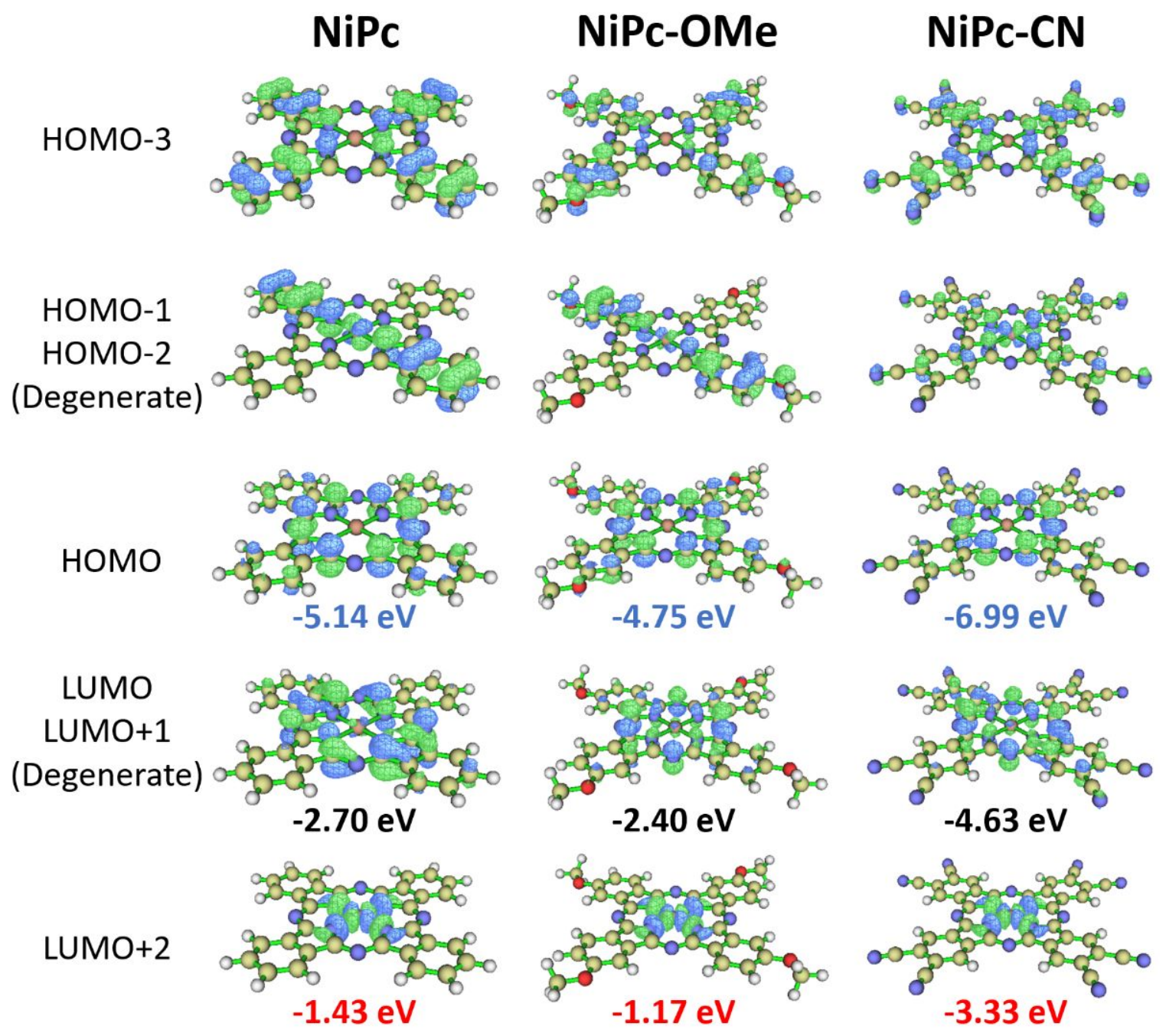

Figure S2. Molecular orbitals of NiPc, NiPc-OMe, and NiPc-CN from their HOMO-3 to LUMO+2 visualized at isosurfaces of 0.04 . The energy levels of selected MOs are labeled under their structural models. Color code: $\mathrm{H}$ - white, $\mathrm{C}$ - tan, $\mathrm{N}$ - blue, $\mathrm{O}$ - red, $\mathrm{Ni}$ - brown. 


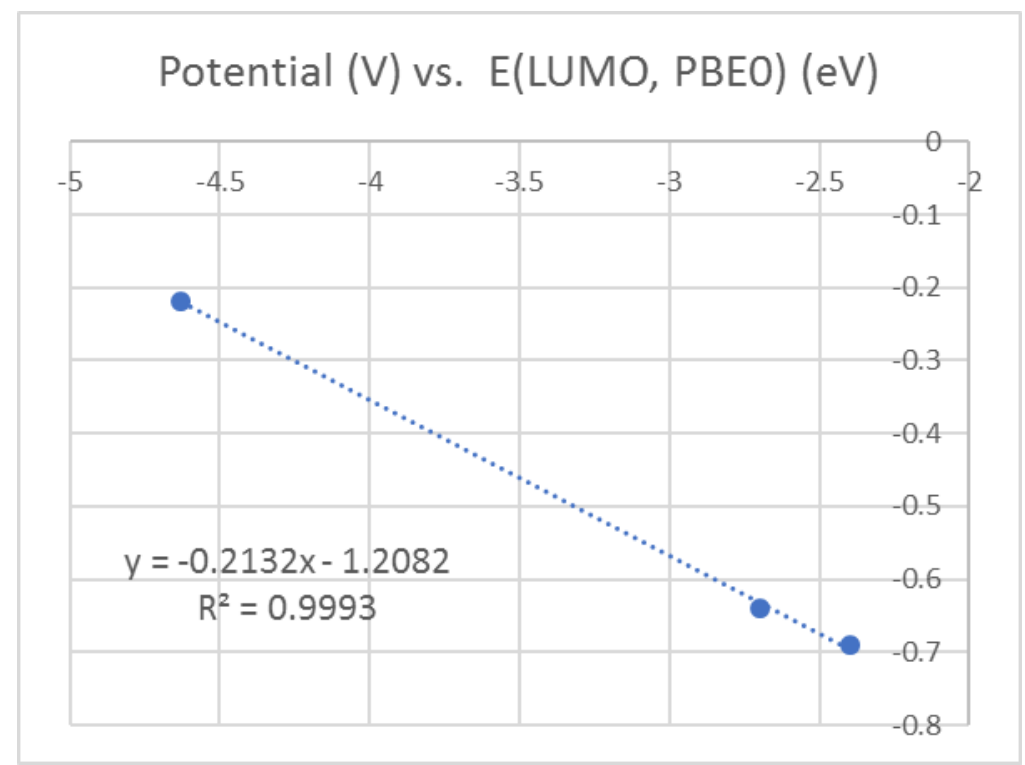

Figure S3. Plot of experimental reduction potential for activation of NiPc/CNT electrocatalysts versus the LUMO energy level of corresponding NiPc molecules calculated at PBE0/def2-SVP, on the set of experimentally reported molecules. Fitted linear relationship is shown in dotted lines, and the fitted formula and $\mathrm{R}$ square value are labeled in the plot. 


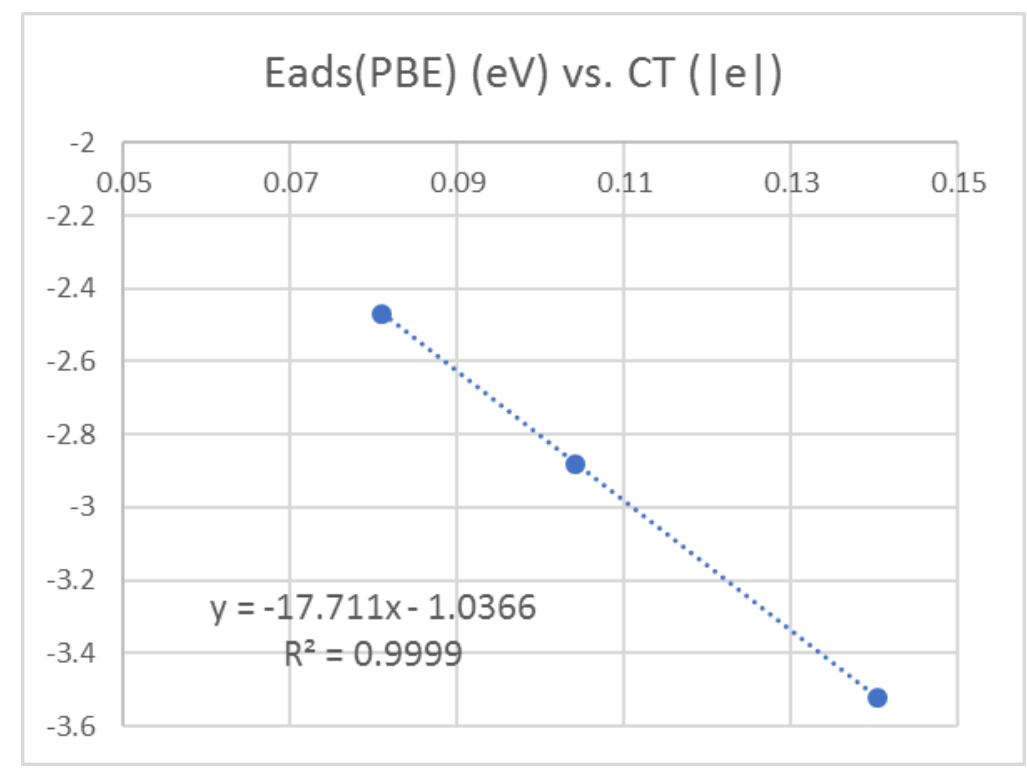

Figure S4. Plot of adsorption energies of NiPc molecules on graphene substrate calculated at PBE-D3 level versus the number of electron transfer to NiPc molecules from surface charging (Figure 7d) calculated at PBE-D3 level, on the set of experimentally reported molecules. Fitted linear relationship is shown in dotted lines, and the fitted formula and $\mathrm{R}$ square value are labeled in the plot. 

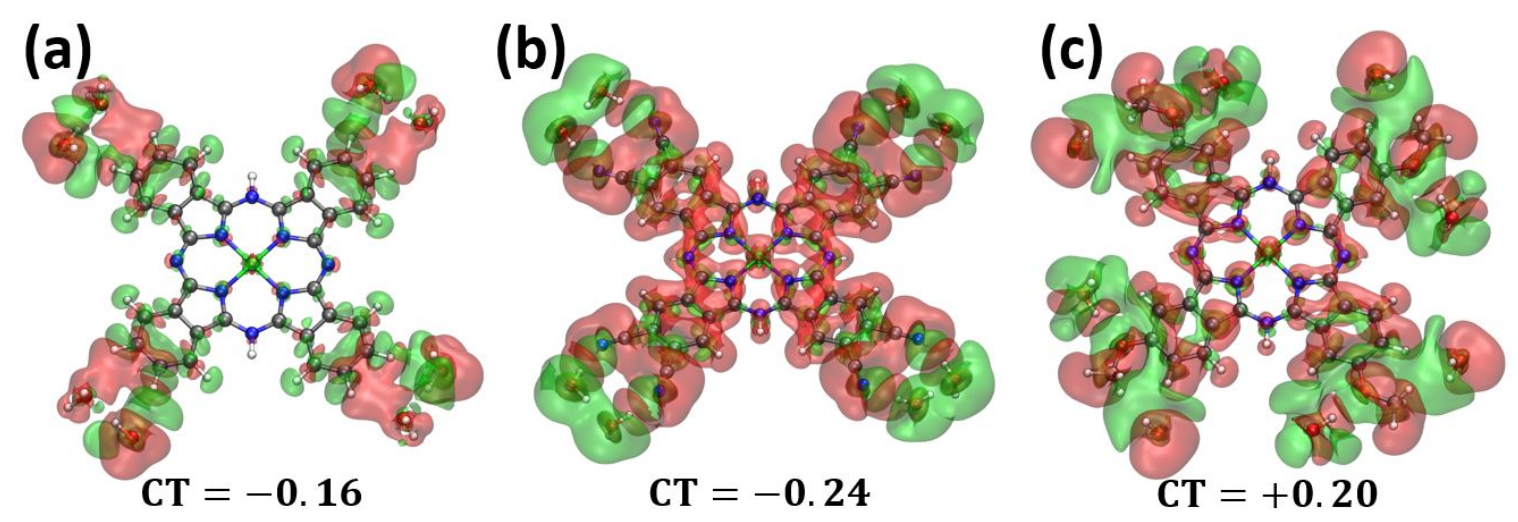

Figure S5. Electron density difference map of (a) NiPc-2H, (b) NiPc-CN-2H, and (c) NiPc-OMe$2 \mathrm{H}$ interacting with 8 explicit water molecules in the periphery, at the isovalue of 0.0001 . The green or red isosurfaces represents electron density increase or decrease, respectively. The net charge transfer from the solvent to the molecular catalyst in labeled below each. 


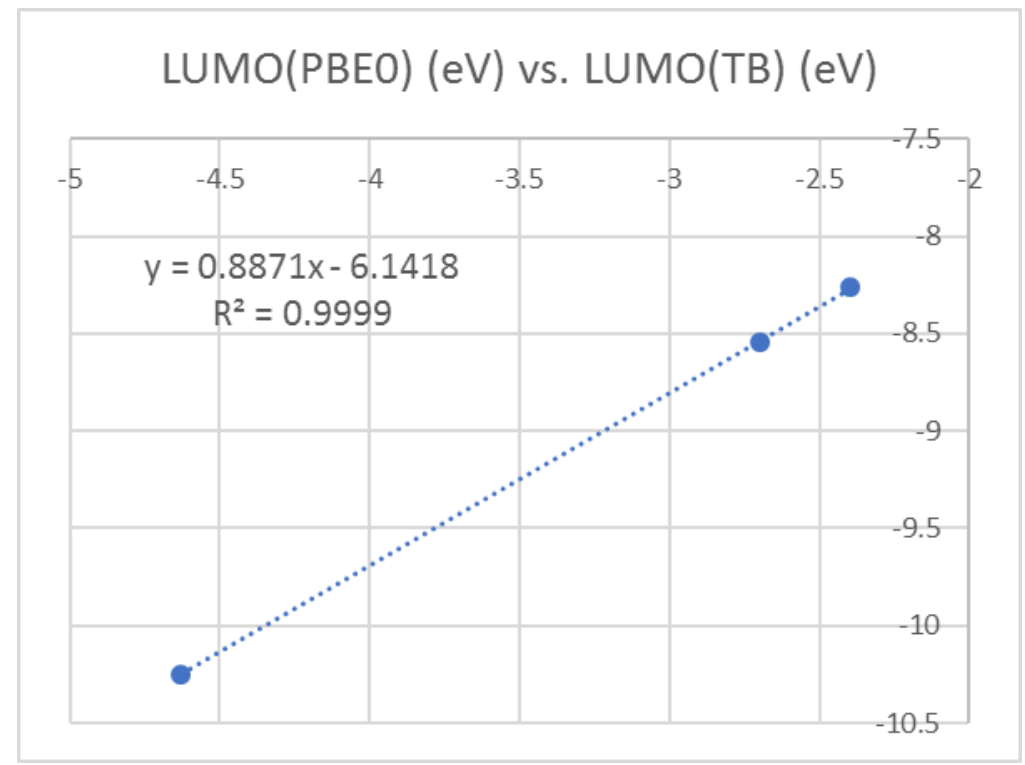

Figure S6. Plot of LUMO energy levels of NiPc molecules calculated at PBE0/def2-SVP versus ones calculated at GFN2-xTB level, on the set of experimentally reported molecules. Fitted linear relationship is shown in dotted lines, and the fitted formula and $\mathrm{R}$ square value are labeled in the plot. 
(a)
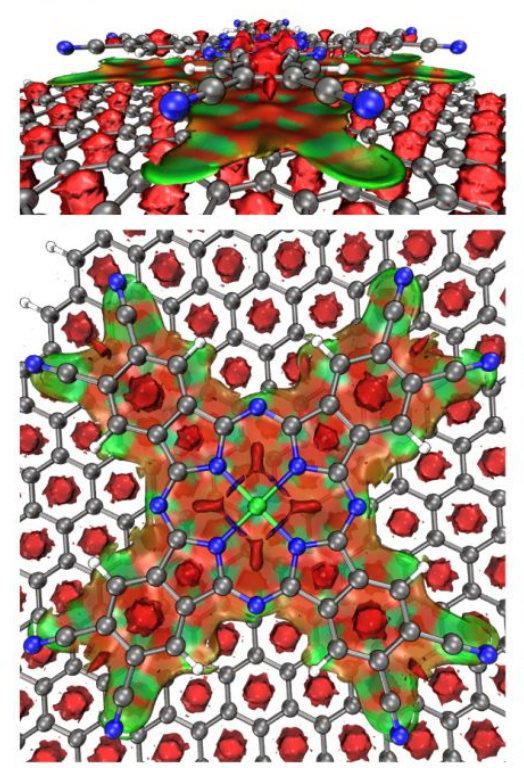

(b)
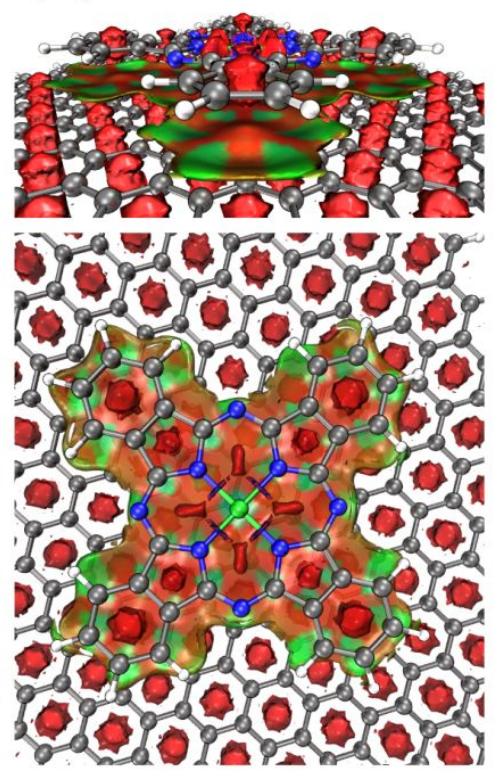

(c)
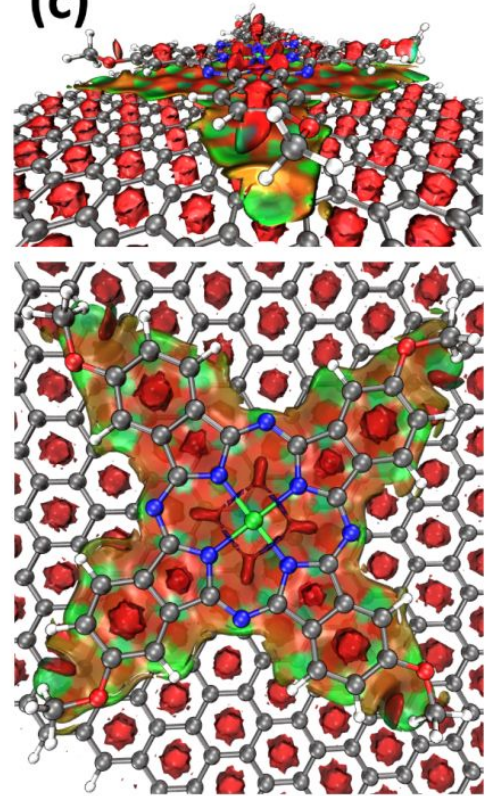

Figure S7. The reduced density gradient isosurfaces from promolecular density showing noncovalent interaction between adsorbed (a) NiPc-CN, (b) NiPc, and (c) NiPc-OMe on aperiodic graphitic carbon sheet calculated at GFN2-xTB. Red and green regions on isosurfaces correspond to repulsive and attractive noncovalent interactions, respectively. Color code: $\mathrm{H}$ - white, $\mathrm{C}$ - gray, $\mathrm{N}$ - blue, $\mathrm{O}$ - red, $\mathrm{Ni}$ - green. 


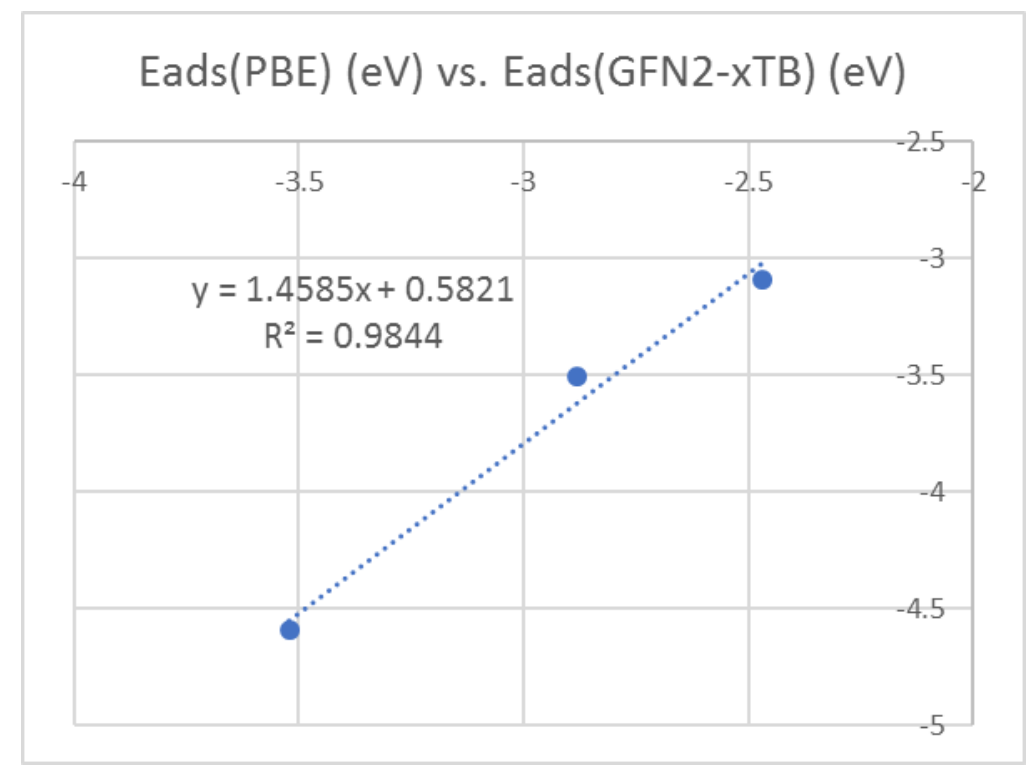

Figure S8. Plot of adsorption energies of NiPc molecules on graphene substrate calculated at PBE-D3 level and adsorption energies of NiPc molecules on an aperiodic graphitic carbon sheet calculated at GFN2-xTB level, on the set of experimentally reported molecules. Fitted linear relationship is shown in dotted lines, and the fitted formula and $\mathrm{R}$ square value are labeled in the plot. 

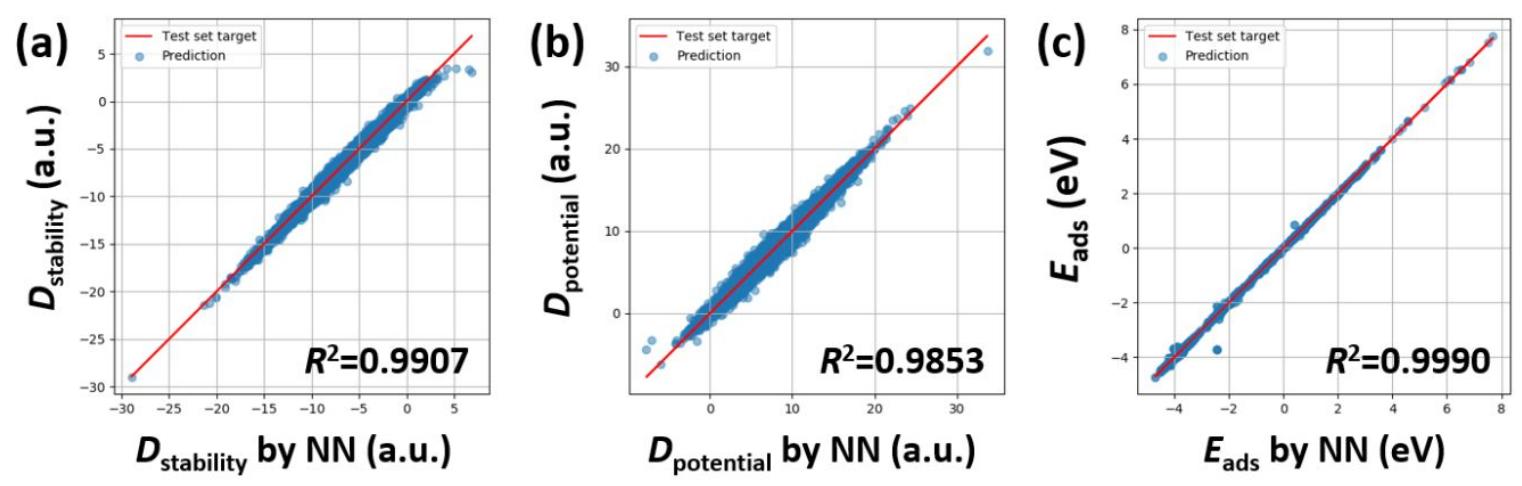

Figure S9. Validation of the trained NN on the test set (randomly chosen $20 \%$ of the data set) for prediction of (a) $D_{\text {stability }}$, (b) $D_{\text {potential, }}$ (c) $E_{\text {ads }}$, with R square labeled in each plot. 


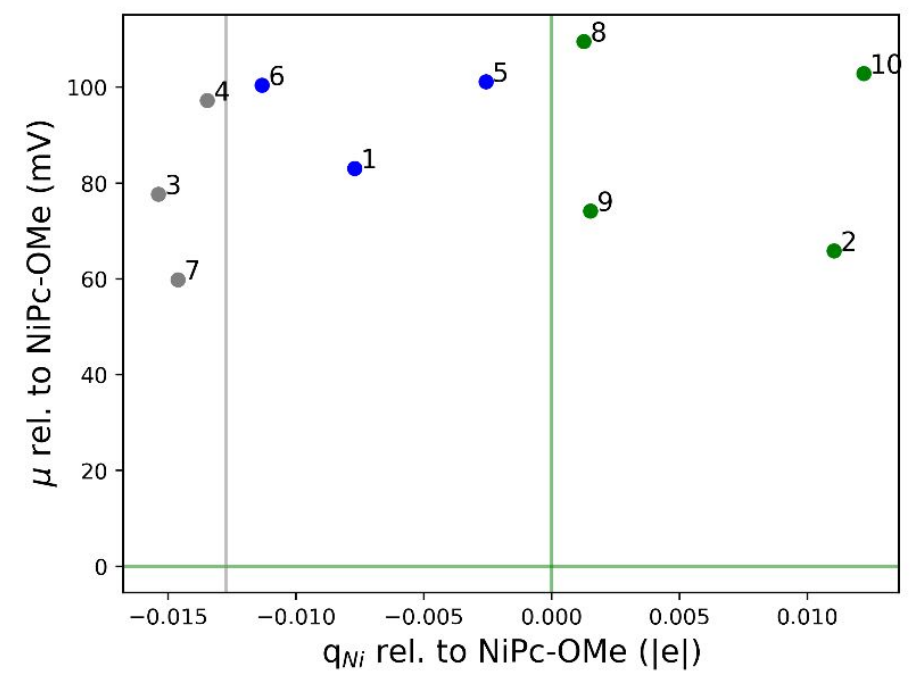

Figure S10. The DFT-calculated reduction potential and Ni Mulliken charges of the ten topscoring candidates from the GA search, relative to the reference system NiPc-OMe. The green and gray lines mark the values for NiPc-OMe and NiPc, respectively. 